\title{
Perinephric Haematoma Causing Refractory Hypertension in a 17-Year-Old Male
}

\author{
Y.P. Kelly J.A. Eustace \\ Department of Nephrology, Cork University Hospital, Cork, Ireland
}

\section{Key Words}

Hypertension - Refractory hypertension - Sympathetic denervation - Renal trauma Hyperreninaemia - Renal haematoma

\begin{abstract}
New-onset arterial hypertension is a well-recognised complication of kidney trauma. Cases have been described in young healthy athletes with hypertension arising years after sportsrelated trauma. The pathophysiology of this disease is thought to arise from intrarenal arterial stenosis resulting from rapid deceleration during the initial injury. This leads to arterial obstruction and ischaemia with increased secretion of renin, eventually leading to elevated blood pressure. Though hypertension in these cases is generally gradual in onset and long-standing, it can also rise acutely, leading to malignant hypertension. We present the case of a 17-year-old male who presented with refractory hypertension following blunt trauma to his left kidney during a recent sporting injury. This is followed by a discussion of the relevant literature in this area to date, highlighting the key challenges involved in the management of these patients.
\end{abstract}

\section{Background}

New-onset arterial hypertension is a well-recognised complication of kidney trauma [1]. The causative mechanism was first described by Page [2] and Engel and Page [3] in the 1940s and can be explained by hyperreninaemia caused by renal compression. Cases have been described in young healthy athletes with new-onset hypertension arising several years after sports-related trauma [4]. Other common preceding events include motor vehicle accidents, mass lesions and renal allograft surgery $[5,6]$. Hypertension in these cases is generally long-standing but can rise acutely, causing malignant hypertension [7]. We present the case of a 17-year-old male who presented with refractory hypertension following blunt trauma to his left kidney during a recent 
sporting injury. This is followed by a discussion of the relevant literature in this area to date.

\section{Case Report}

A 17-year-old male presented to the emergency department of a university teaching hospital following an episode of headache, dizziness, confusion and disorientation which was gradual in onset while playing a hurling match. He had not sustained any trauma during the game. Blood pressure on arrival measured 157/90 mm Hg. On auscultation of his chest, a systolic murmur was appreciated, maximal in the aortic area. Fundoscopy was not reported. Brain CT and lumbar puncture showed no abnormality. Urea and electrolytes were not deranged, and a chest X-ray was unremarkable, with no evident rib notching. An ECG revealed a left ventricular strain pattern. Echocardiography was normal apart from mild anterior mitral valve leaflet prolapse with mild mitral regurgitation. His symptoms were attributed to viral illness, and the patient was discharged with follow-up arranged for a review clinic.

He subsequently represented 1 week later with persistent headache and elevated blood pressure, ranging between 137-154/75-89 mm Hg on testing by his GP. Clinical examination was unchanged. Subsequent questioning revealed that the patient had sustained a blow to his left flank during a hurling match 2 weeks earlier. He had not sought medical assessment at that time and was able to continue playing the match. However, the symptoms were significant enough for him to mention them to his parents afterwards. The flank tenderness settled spontaneously without any intervention. Urinalysis was negative for any abnormality, and 24-hour urinary metanephrine screening was negative on two occasions. Serum renin-aldosterone ratio was normal at 0.9, despite raised renin to $77.3 \mathrm{ng} / \mathrm{l}$. A CT renal angiogram was performed which showed a large haematoma overlying the left kidney, with no evidence of renal atrophy or renal artery stenosis (fig. 1, fig. 2 fig. 3 ). The adrenal glands were normal in appearance. Urinalysis was negative for haematuria and proteinuria.

He was commenced on bisoprolol $2.5 \mathrm{mg}$. Blood pressure settled only to $150 / 80 \mathrm{~mm} \mathrm{Hg}$ so this was switched to ramipril $2.5 \mathrm{mg}$. Stepwise increments of his anti-hypertensive medications were required to achieve control of his elevated blood pressure until ultimate stability was achieved with 4 agents - amlodipine, ramipril, valsartan and spironolactone - reaching a blood pressure of 120/60 $\mathrm{mm}$ Hg. A CT-guided aspiration of the left perinephric haematoma was then attempted but this unfortunately was unsuccessful as the fluid collection had become organised. He remained asymptomatic and normotensive on therapy over the next 3 months, and repeat electrocardiography showed reversal of the left ventricular hypertrophy and strain pattern previously seen. Repeat renal ultrasound later showed substantial reduction in the thickness of the left-sided perinephric crescentic collection to $<1 \mathrm{~cm}$ ( $\underline{\text { fig. }}$ ). Vascularity was symmetric, and both kidneys measured $11 \mathrm{~cm}$ in size. The young boy unfortunately remains on 4 anti-hypertensive agents to date at 1 year of follow-up.

\section{Discussion}

Blunt renal trauma has become well recognised as a cause of secondary hypertension, with an incidence as high as $40 \%$ in one retrospective review of a level 1 trauma database over 20 years [1]. Better protective equipment reduces the risk of this complication. Other related causes include renal allograft haematomas, renal biopsies, mass lesions, anticoagulation, vasculitis, lithotripsy, sympathetic nerve block and pyeloplasty. Our hypothesis in this case is that blunt trauma to the left kidney at the time of the initial injury caused a haematoma, possibly arising from an underlying arteriovenous malformation or another congenital anomaly in the renal vasculature. This haematoma later expanded and caused symptomatic accelerated hypertension while the patient subsequently played in a further sporting event. Definitive testing for vascular malformations in this setting requires focused imaging with renal 
angiography. This, however, has not been performed to date in this case as the young boy's haematoma resorbed satisfactorily with conservative management. This decision may need to be re-addressed in the future if hypertension fails to settle to allow the gradual withdrawal of the anti-hypertensive agents.

The pathology of hypertension related to renal trauma is characterised by intrarenal artery or segmental branch stenosis [8]. This may result from rapid deceleration which injures the renal arterial intima, leading to obstruction and ischaemia. Collateralisation of renal blood supply subsequently arises, which preserves renal endocrine function and leads to an increase in renin secretion, eventually causing hypertension [4, 9]. Sympathetic nerve system activation also plays a dominant role in promoting and perpetuating hypertension following renal trauma. Its mechanism has been further elucidated through recent advances in quantification through biochemical, neurophysiological and bioengineering methods [10]. Catheter-based radiofrequency ablation of bilateral renal nerves has further supported the importance of afferent and efferent renal sympathetic nerves in the pathogenesis of hypertension in these cases. Sustained medium-term blood pressure-lowering effects have been demonstrated for patients with resistant hypertension using this procedure. To date, however, this method has only been utilised in open-label trials in highly selected patient groups with refractory hypertension and suitable vascular anatomy, hence its risk-benefit ratio in the long-term for routine practice remains elusive at present [11].

Blood pressure may rise acutely, resulting in malignant hypertension [7], as in this case. This may be attributed to increased renal nerve activity dating from the time of injury [12]. Outcomes can vary widely as in some cases hypertension resolves spontaneously while becoming refractory to treatment in other settings. Many cases are entirely asymptomatic and may only be picked up by renal atrophy on later imaging. In symptomatic cases, lumbar pain is a presenting complaint in $50 \%$ of patients [13]. Serum electrolyte levels are usually normal, but hypokalaemia $(<3.5$ $\mathrm{mEq} / \mathrm{l}$ ) is thought to act as a risk factor for the development of malignant hypertension [13]. This was not, however, a notable feature in our case. Diagnosis is usually made by CT or ultrasonography $[14,15]$, with CT thought to allow greater anatomic accuracy. MRI is useful for differentiating urinomas from haematomas and aging haematomas. Selective renal arteriography with renal vein renin assays is the gold standard as it identifies lesions in the renal vasculature and couples this with confirming their functional significance, namely through comparing the ratio of elevated renin from the damaged kidney to suppressed renin from the contralateral kidney.

Patients should be treated to control hypertension and renal parenchymal damage. Irreversible structural damage is likely to have occurred if hypertension does not resolve within 1-2 years [1]. Anti-hypertensives are used which block the reninangiotensin-aldosterone axis, usually ACE inhibitors and angiotensin receptor blockers $[4,5]$, in conjunction with a diuretic. Direct renin inhibitors are now also available but have not yet been studied in this circumstance [16]. Surgical intervention is usually dependent on the size of the haematoma and associated kidney damage. Early intervention is considered in cases where hypertension worsens with evidence of endorgan damage or with the occurrence of significant medication side effects [5]. The range of procedures include percutaneous drainage of small haematomas [14] as in our case, capsulectomy for fibrotic and adherent clots [17], laparoscopic/open evacuation of haematomas or radical nephrectomy for extensive damage. 


\section{Conclusions}

Accelerated poorly controlled hypertension occurs relatively commonly following blunt renal trauma/intervention causing perinephric haematoma and renal compression. It requires early diagnosis and treatment to avoid long-term complications and damage for patients, as they tend to be generally young and otherwise healthy. Resulting renal parenchymal scarring and uncontrolled hypertension if not identified and treated may exert major health and financial consequences. The significant role played by early and adequate history taking is highlighted in the above case as this was crucial to focus effective investigations and management decisions for this young patient. The performance of fundoscopy to check for papilloedema is also noted as a key focus for clinical examination.

Relevant investigations should include blood tests to evaluate the renin-angiotensinaldosterone system as well as appropriate imaging. Medical management is used initially, with a possible growing role for direct renin inhibitors as monotherapy in this regard. Surgery also plays an important role in deteriorating cases as well as in those with a reversible mechanical obstruction. Catheter radiofrequency renal nerve ablation may become a useful primary or adjunctive therapy for hypertension control in patients with suitable anatomy. Relatively unusual features of this case include the rapidity of the onset of hypertension after injury and the refractory nature of the disease for this young patient, causing early evidence of end-organ damage, which was later reversible with appropriate treatment. This case explores the various diagnostic and therapeutic options which are currently advocated and emphasises the importance of their timeliness in promoting a superior outcome for the patient. 


\section{Case Reports in Nephrology and

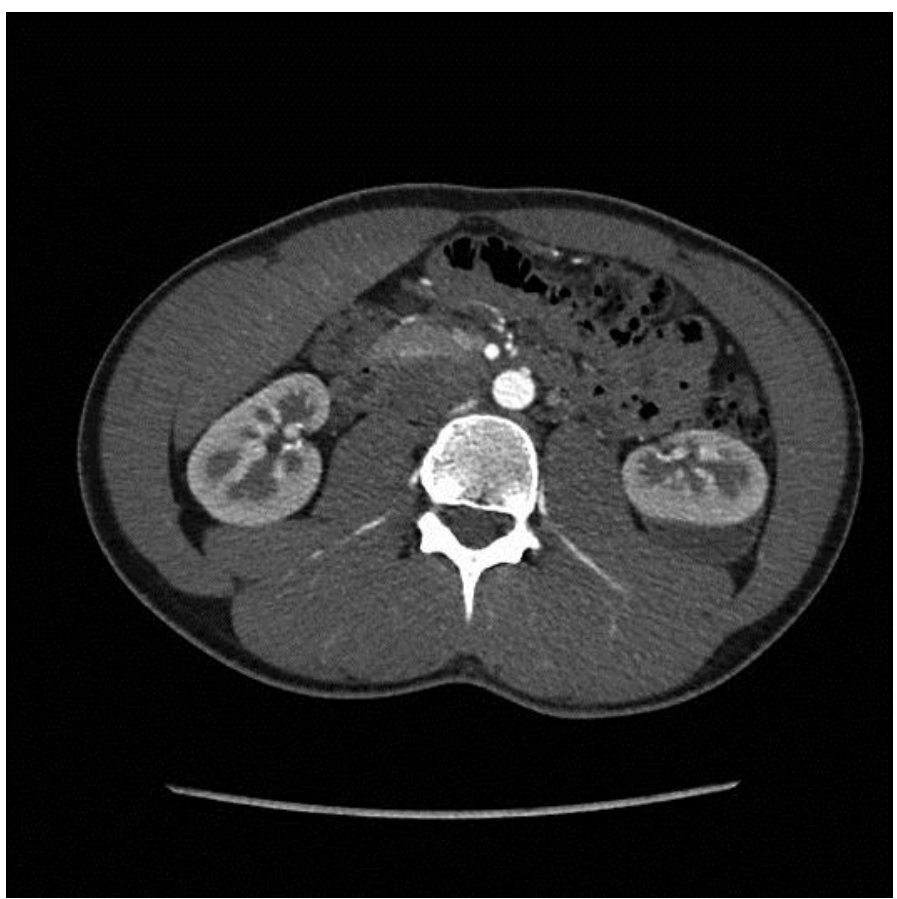

Fig. 1. Transverse slice of the CT renal angiogram depicting the perinephric haematoma at the inferior pole of the left kidney.

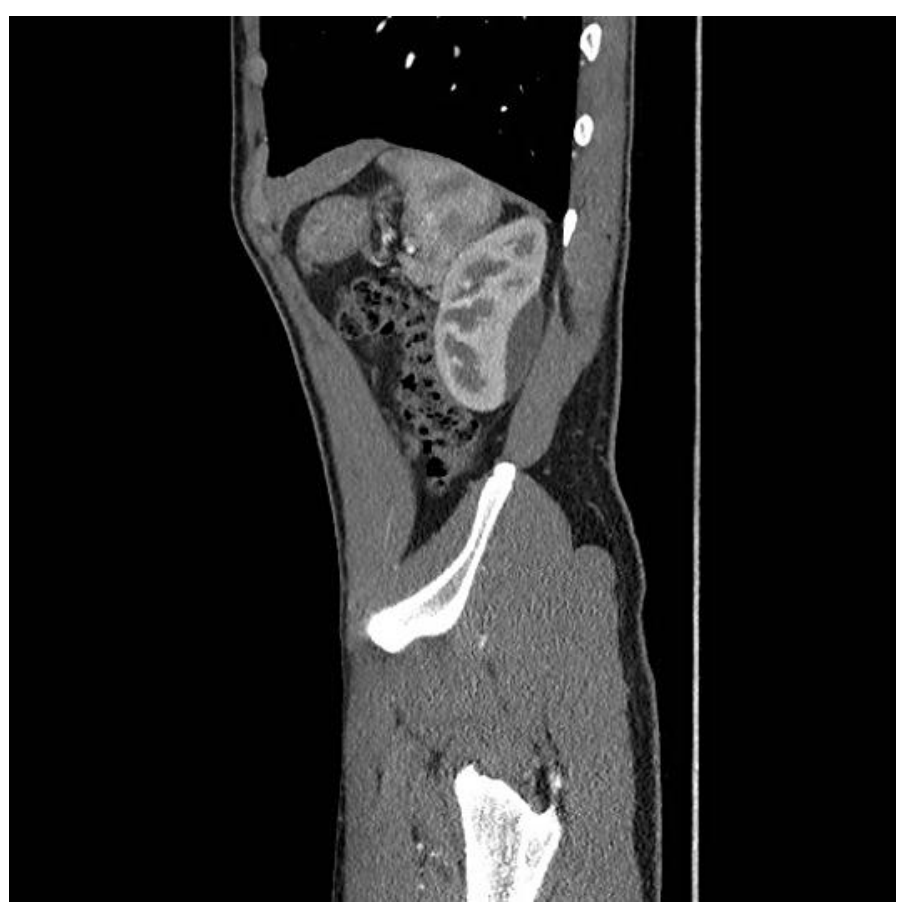

Fig. 2. Sagittal slice of the CT renal angiogram depicting the perinephric haematoma at the inferior pole of the left kidney. 


\section{Case Reports in Nephrology and

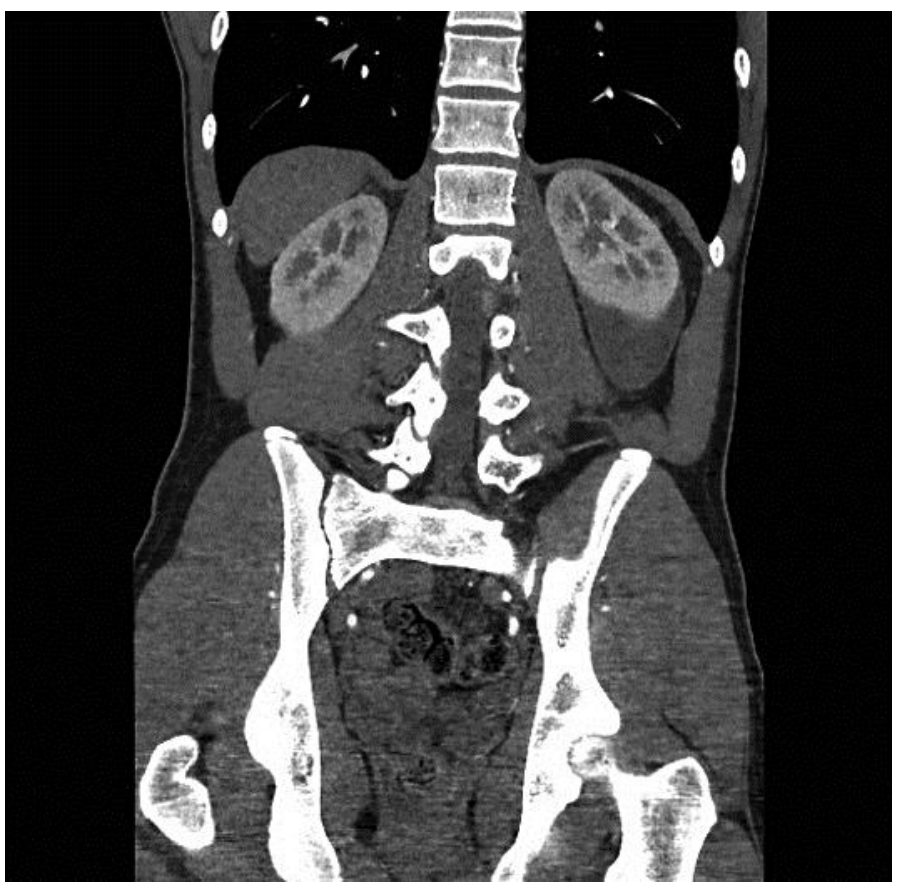

Fig. 3. Coronal slice of the CT renal angiogram depicting the perinephric haematoma at the inferior pole of the left kidney.

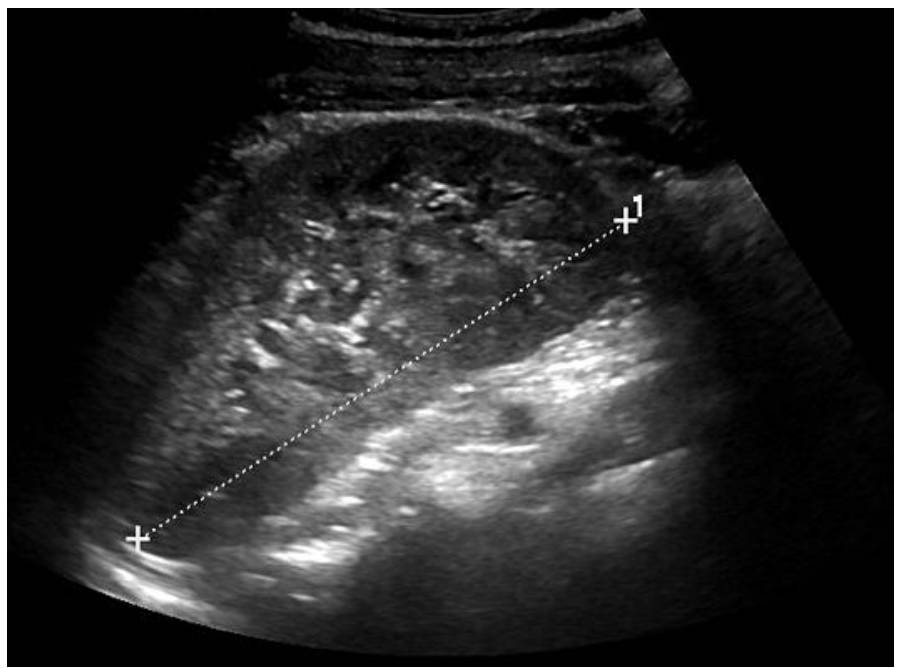

Fig. 4. Interval renal ultrasound at 6 months after injury showing almost complete resolution of the left perinephric haematoma. 


\section{References}

1 Montgomery RC, Richardson JD, Harty JI: Posttraumatic renovascular hypertension after occult renal injury. J Trauma 1998;45:106-110.

-2 Page IH: Arterial hypertension 1942. Conn Med 1992;56:455-456.

-3 Engel WJ, Page IH: Hypertension due to renal compression resulting from subcapsular hematoma. J Urol 1955;73:735-739.

4 McCune TR, Stone WJ, Brever JA: Page kidney: case report and review of the literature. Am J Kidney Dis 1991;18:593-599.

5 Dopson SJ, Jayakumar S, Velez JC: Page kidney as a rare cause of hypertension: case report and review of the literature. Am J Kidney Dis 2009;54:334-339.

-6 Yung BC, Wong KW, Fan WC, Chan JC, Lo SS: Negative captopril renography on patients with renin mediated hypertension due to page kidney and reninoma. Eur J Radiol 1999;31:63-68.

7 Paris B, Bobrie G, Rossignol, Le Coz S, Chedid A, Plouin PF: Blood pressure and renal outcomes in patients with kidney infarction and hypertension. J Hypertens 2006;24:1649-1654.

8 Carroll PR, McAninch JW, Klosterman P, Greenblatt M: Renovascular trauma: risk assessment, surgical management and outcome. J Trauma 1990;30:547-552.

-9 Sterns RH, Rabinowitz R, Segal AJ, Spitzer RM: Page kidney hypertension caused by chronic subcapsular haematoma. Arch Intern Med 1985;145:169-171.

10 Grassi G, Bertoli S, Seravalle G: Sympathetic nervous system: role in hypertension and in chronic kidney disease. Curr Opin Nephrol Hypertens 2012;21:46-51.

11 Azizi M, Steichen O, Frank M, Bobrie G, Plouin F, Sapoval M: Catheter-based radiofrequency renal-nerve ablation in patients with resistant hypertension. Eur J Vasc Endovasc Surg 2012;43:293-299.

12 Herlitz H, Hjemdahl P, Delin K, Granerus G, Aurell M: Plasma noradrenaline and dopamine in renin-mediated hypertension. Clin Physiol 1990;10:27-36.

13 John J, Allen S, Perry M, Patel MR, O’Brien T: Page kidney phenomenon presenting as acute renal failure after partial nephrectomy: a case report and review of the literature. Urol Int 2008;80:440-443.

14 Matlaga BR, Veys JA, Jung F, Hutcheson J: Subcapsular urinoma: an unusual form of Page kidney in a high school wrestler. J Urol 2002;168:672.

15 Diamond JA: Hypertension due to perinephric compression: the 'Page' kidney. Am J Hypertens 2001;14:305.

16 Abassi Z, Armaly Z, Nakhoul F, Hoffman A: Oral inhibitors of renin and their potential use as therapeutic agents in treating hypertension (in Hebrew). Harefuah 2008;147:536-542.

17 Moriarty KP, Lipkowitz GS, Germain MJ: Capsulectomy: a cure for the Page kidney. J Pediatr Surg 1997;32:831-833. 